\title{
Visualizing Convolutional Neural Networks with Virtual Reality
}

\author{
Nadine Meissler \\ German Aerospace Center \\ Cologne, Germany \\ nadine.meissler@dlr.de
}

\author{
Annika Wohlan \\ German Aerospace Center \\ Cologne, Germany \\ annika.wohlan@dlr.de
}

\author{
Nico Hochgeschwender \\ German Aerospace Center \\ Cologne, Germany \\ nico.hochgeschwender@dlr.de
}

\begin{abstract}
Software systems and components are increasingly based on machine learning methods, such as Convolutional Neural Networks (CNNs). Thus, there is a growing need for common programmers and machine learning newcomers to understand the general functioning of these algorithms. However, as neural networks are complex in nature, novel presentation means are required to enable rapid access to the functionality. For that purpose, this paper examines how CNNs can be visualized in Virtual Reality. A first exploratory study has confirmed that our visualization approach is both intuitive to use and conductive to learning.
\end{abstract}

\section{CCS CONCEPTS}

- Computer methodologies $\rightarrow$ Virtual Reality; Neural networks.

\section{KEYWORDS}

neural networks, visualization, virtual reality, knowledge learning

\section{ACM Reference Format:}

Nadine Meissler, Annika Wohlan, and Nico Hochgeschwender. 2019. Visualizing Convolutional Neural Networks with Virtual Reality. In 25th ACM Symposium on Virtual Reality Software and Technology (VRST'19), November 12-15, 2019, Parramatta, NSW, Australia. ACM, New York, NY, USA, 2 pages. https://doi.org/10.1145/3359996.3364817

\section{INTRODUCTION}

Deep neural network architectures have demonstrated tremendous performance in prediction and classification tasks on a various sort of data such as images, text and speech, and video data. However, given the size and complexity of non-trivial networks, they are often considered as black-box function approximators. While explainability, interpretability and transparency of neural networks is an own research field, [Chakraborty et al. 2017] we aim to enable non-experts to obtain a basic understanding of the functionality of neural networks. To do so, we present and evaluate a visualization concept of Convolutional Neural Networks (CNN) in Virtual Reality (VR). VR with head-mounted displays has proven itself as a learning medium in the engineering field [Abulrub et al. 2011]. Indeed, there is evidence that a virtual learning environment can be able to achieve better learning outcomes than traditional teaching [Alhalabi 2016]. Our main goal is to get insights in whether the use of virtual reality technology enables visualization possibilities

Permission to make digital or hard copies of part or all of this work for personal or classroom use is granted without fee provided that copies are not made or distributed for profit or commercial advantage and that copies bear this notice and the full citation on the first page. Copyrights for third-party components of this work must be honored. For all other uses, contact the owner/author(s).

VRST '19, November 12-15, 2019, Parramatta, NSW, Australia

(C) 2019 Copyright held by the owner/author(s).

ACM ISBN 978-1-4503-7001-1/19/11.

https://doi.org/10.1145/3359996.3364817 that facilitate the learning process of understanding artificial neural networks.

\section{NEURAL NETWORK VISUALIZATION}

Previous visualization approaches for neural networks are either tailored to developers of machine learning systems (e.g., TensorFlow's visualization toolkit TENSORBOARD ${ }^{1}$ ) or require detailed prior knowledge, such as an understanding of the underlying learning processes or significant cognitive effort [Yosinski et al. 2015]. There are a few approaches to convey complex concepts, such as CNNs via VR, as users perceive virtual worlds as attractive learning environments [Dede et al. 1996]. For instance, Bock et al.[Schreiber and Bock 2019] developed a 3D visualization of deep learning algorithms for experts and non-experts with an interactive user interface that allows interactive exploration on different levels of detail. However, this concept has not yet been implemented and evaluated in a virtual environment.

\subsection{Convolutional Neural Network}

Our visualization is intended to illustrate the structure and functionality of a CNN in VR (see Figure 1). The model that we visualize is defined in Keras using Python. For testing and evaluation, we use a simple CNN model that is based on an example in the KerAs documentation [Chollet et al. 2015]. Our visualization of filter and feature maps is based on a methodology described by Irvine [Irvine 2017]. Feature maps of each convolutional layer and each pooling layer are rendered as 2D images with MATPLOTLIB using the gray colormap.

The user is able to select different input images and have them classified by CNN. For each different layer of the CNN, users see intermediate results for the respective input image and the output of the model. A interactive 3D-model is the main anchor point of the visualisation and therefore placed in Area 1. It models the structure of the $\mathrm{CNN}$ on the basis of the individual layers. The representation is based on the 2D-representation of LeNet-5 [LeCun et al. 1998]. Area 2 displays the main information for the selected layer, divided in input, operation and output of the layer. For the convolutional layer it displays a visualization of the filters and the produced feature maps (Area 3). Area 4 offers additional information to specific items of Area 2. Area 5 functions as an edition to the 3D-model and provides further details on the parameters chosen for the operations. In addition, it highlights the dimensions of the input and output data of the specific operation.

\subsection{Exploratory Study}

We executed an exploratory study with 14 computer scientists $\left(\mathrm{M}_{\text {age }}\right.$ $\left.=28.29, \mathrm{SD}_{\text {age }}=4.41\right)$ to gather insights into how users would use

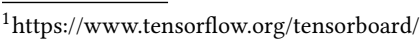




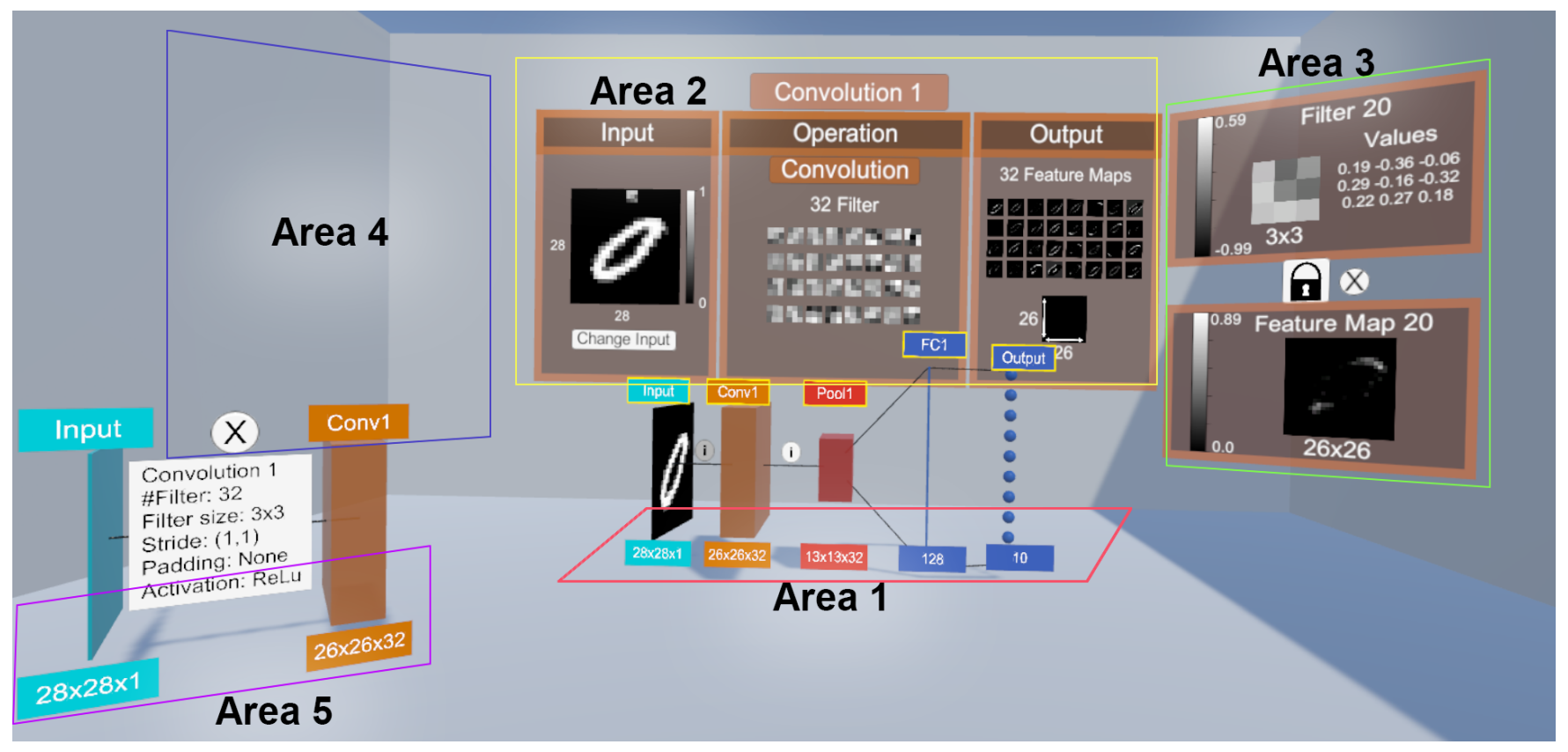

Figure 1: Overview of the five visual areas of the $\mathrm{CNN}$ visualization.

and evaluate our visualization approach. Before entering the VR, participants were instructed to read an introductory text on CNNs. Next, participants had about 10 minutes in VR to study CNNs. There were no specific instructions for interacting with the visualization. Afterwards, they were asked about satisfaction, perceived learning success, and perceived usefulness.

In general, the participants claimed that our visualization has a positive impact on learning. This is reflected by their improvement of knowledge (see Figure 2). Our study also confirms that our visualization approach is both intuitive and engaging.

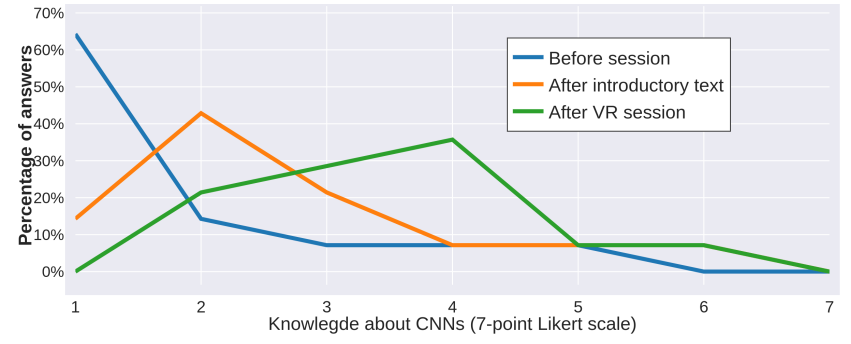

Figure 2: Learning effect: Participants' self-reported knowledge about $\mathrm{CNN}$ before user session, after reading the introductory text, and after experiencing the VR visualization.

\section{CONCLUSION AND FUTURE WORK}

We present a first approach to visualize CNNs in VR with the aim to give users an introduction to its functionality. We propose that immersive VR has the potential to facilitate learning about architecture of CNNs. The results of our exploratory study indicate that VR provide a stable learning environment for studying CNNs. However, we cannot say whether VR enables a better learning experience than traditional learning. Thus, we plan to create an equivalent prototype of this visualization for a traditional desktop visualization. A follow up quantitative user study, comparing VR with desktop $3 \mathrm{D}$, will provide further information.

\section{REFERENCES}

A. G. Abulrub, A. N. Attridge, and M. A. Williams. 2011. Virtual Reality in engineering education: The future fo creative learning. 2011 IEEE Global Engineering Education Conference (EDUCON) (2011), 751-757. https://doi.org/10.1109/EDUCON.2011. 5773223

Wadee Alhalabi. 2016. Virtual reality systems enhance students' achievements in engineering education. Behaviour \& Information Technology 35, 11 (July 2016), 919-925. https://doi.org/10.1080/0144929X.2016.1212931.

S. Chakraborty, R. Tomsett, R. Raghavendra, D. Harborne, M. Alzantot, F. Cerutti, M. Srivastava, A. Preece, S. Julier, R. M. Rao, T. D. Kelley, D. Braines, M. Sensoy, C. J. Willis, and P. Gurram. 2017. Interpretability of deep learning models: A survey of results. In 2017 IEEE SmartWorld, Ubiquitous Intelligence Computing, Advanced Trusted Computed, Scalable Computing Communications, Cloud Big Data Computing, Internet of People and Smart City Innovation (SmartWorld/SCALCOM/UIC/ATC/CBDCom/IOP/SCI). 1-6. https://doi.org/10.1109/UICATC.2017.8397411

François Chollet et al. 2015. Keras. https://keras.io.

C. Dede, M. C. Salzman, and R. B. Loftin. 1996. ScienceSpace: Virtual realities for learning complex and abstract scientific concepts., In Proceedings of the IEEE 1996 Virtual Reality Annual International Symposium. Proceedings of the IEEE 1996 Virtual Reality Annual International Symposium, 246-252. https://doi.org/10.1109/ VRAIS.1996.490534

Mike Irvine. 2017. Introduction to Convolutional Neural Networks. (2017). https: //sempwn.github.io/blog/2017/04/06/conv_net_intro

Yan LeCun, Leon Bottou, Yoshua Bengio, and Patrick Haffner. 1998. Gradient-Based Learning applied to document recognition. Proc. IEEE 86, 11 (1998), $2278-2324$. https://doi.org/10.1109/5.726791

Andreas Schreiber and Marcel Bock. 2019. Visualization and Exploration of Deep Learning Networks in 3D and Virtual Reality. In HCI International 2019 - Posters. Springer International Publishing, 206-211. https://doi.org/10.1007/978-3-03023528-4_29

Jason Yosinski, Jeff Clune, Anh Nguyen, Thomas Fuchs, and Hod Lipson. 2015. Understanding Neural Networks Through Deep Visualization. In In ICML Workshop on Deep Learning. 